\title{
Chinese Society of Interventional Radiology Expert Consensus on the prevention and control of COVID-19 in interventional radiology procedures (first edition)
}

\author{
Yueqi Zhu ${ }^{1 \#}$, Yingkun He ${ }^{2 \#}$, Zhongmin Wang ${ }^{3}$, Zhengyu Jin ${ }^{4}$, Chuansheng Zheng ${ }^{5}$, Gang Sun ${ }^{6}$, Haibin Shi ${ }^{7}$, \\ Hailiang $\mathrm{Li}^{8}$, Xiao $\mathrm{Li}^{9}$, Hua Xiang ${ }^{10}$, Ligong Lu ${ }^{11}$, Shi Zhou ${ }^{12}$, Tianxiao $\mathrm{Li}^{2}$, Xiaoguang $\mathrm{Li}^{13}$, Haidong Zhu ${ }^{14}$, \\ Mingsheng Huang ${ }^{15}$, Feng Duan ${ }^{16}$, Haibo Shao ${ }^{17}$, Tianzhi An ${ }^{12}$, Yingsheng Cheng ${ }^{1}$; Chinese Society of \\ Interventional Radiology
}

${ }^{1}$ Department of Radiology, Shanghai Jiao Tong University Affiliated Sixth People's Hospital, Shanghai 200233, China; ${ }^{2}$ Department of Interventional Neuroradiology, Henan Provincial People's Hospital, Zhengzhou 450003, China; ${ }^{3}$ Department of Radiology, Rui Jin Hospital/Lu Wan Branch, School of Medicine, Shanghai Jiaotong University, Shanghai 200025, China; ${ }^{4}$ Department of Radiology, Peking Union Medical College Hospital, Beijing 100730, China; ${ }^{5}$ Department of Radiology, Union Hospital, Tongji Medical College, Huazhong University of Science and Technology, Wuhan 430022, China; ${ }^{6}$ Department of Radiology, The 960 the Hospital of Joint Logistics Support of PLA, Jinan 250031, China; ${ }^{7}$ Department of Radiology, The First Affiliated Hospital of Nanjing Medical University, Nanjing 210029, China; ${ }^{8}$ Minimally Invasive and Interventional Department, Henan Cancer Hospital, The Affiliated Cancer Hospital of Zhengzhou University, Zhengzhou 450008, China; ${ }^{9}$ Department of Interventional Therapy, National Cancer Center/National Clinical Research Center for Cancer/Cancer Hospital, Chinese Academy of Medical Sciences and Peking Union Medical College 17 Panjiayuan Nanli, Beijing 100021, China; ${ }^{10}$ Department of Interventional Radiology and Vascular Surgery, Hunan Provincial People's Hospital, Changsha 410005, China; ${ }^{11}$ Department of Interventional Radiology, Zhuhai Hospital Affiliated with Jinan University, Zhuhai 519000, China; ${ }^{12}$ Department of Interventional Radiology, The Affiliated Hospital of Guizhou Medical University, Guiyang 550004, China; ${ }^{13}$ Minimally Invasive Tumor Therapies Center, Beijing Hospital, National Center of Gerontology, Institute of Geriatric Medicine, Chinese Academy of Medical Sciences, Beijing 100730, China; ${ }^{14}$ Center of Interventional Radiology and Vascular Surgery, Department of Radiology, Zhongda Hospital, Southeast University, Nanjing 210009, China; ${ }^{15}$ Department of Interventional Radiology, The Third Affiliated Hospital of Sun Yat-sen University, Guangzhou 510000, China; ${ }^{16}$ Department of Interventional Radiology, The General Hospital of Chinese People's Liberation Army, Beijing 100853, China; ${ }^{17}$ Department of Interventional Radiology, The First Hospital of China Medical University, Shenyang 110001 , China

"These authors contributed equally to this work.

Correspondence to: Yingsheng Cheng, MD. Chairmen of the Chinese Society of Interventional Radiology, No. 600, Yishan Road, Shanghai, 200233, China. Email: chengyingsheng@hotmail.com; Chuansheng Zheng, MD. No. 1277, Jiefang Avenue, Wuhan 430022, China. Email: hqzcsxh@sina.com; Zhongmin Wang, MD. No. 197, Second Ruijin Road, Shanghai, 200025, China. Email: wzm0722@hotmail.com.

\begin{abstract}
The COVID-19 pandemic seriously threatens the lives of the general public and poses momentous challenges to all medical workers, including those engaged in interventional radiology, who play an important role in the diagnosis and treatment of various diseases. To further standardize the prevention and control of nosocomial infections and ensure the safety of doctors and patients, the Chinese Society of Interventional Radiology (CSIR) organized multidisciplinary experts in the field of interventional radiology in China to prepare an "Expert Consensus" elaborating and summarizing the protective strategies and suggestions for medical workers in the field of interventional radiology when they engage in interventional diagnosis and treatment activities against the background of novel coronavirus infection control. The aim is to provide a reference for interventional procedures in hospitals and other medical institutions at all levels in China and worldwide. The key points include the following: (I) non-emergency interventional diagnosis and treatment should be suspended while work is ongoing to prevent and control the spread of COVID-19; (II) protective measures should be taken according to the appropriate level designated for COVID-19 infection prevention and control; (III) patients should take measures to protect themselves when they want to see a doctor, including accessing outpatient services online and other relevant channels of consultation.
\end{abstract}


Keywords: COVID-19; pneumonia; interventional radiology

Submitted Apr 01, 2020. Accepted for publication Apr 24, 2020.

doi: 10.21037/qims.2020.04.11

View this article at: http://dx.doi.org/10.21037/qims.2020.04.11

\section{Introduction}

At the end of December 2019, numerous cases of pneumonia of unknown aetiology appeared in Wuhan $(1,2)$. Subsequent viral gene sequencing revealed that the pathogen was a new type of coronavirus (3-5). The National Health Commission of China named it novel coronavirus disease, or COVID-19, which is consistent with the naming conventions of the World Health Organization (6). In a short period of time, there has been a significant increase in the number of infections in China and around the world, with 81,518 COVID-19 infections in China, 785,979 infections worldwide, and a total of 37,810 deaths as of March 31, 2020 (7) (Figure 1). On January 20, 2020, China's National Health Commission categorized COVID-19 as a class B infectious disease, as stipulated in the "Law of the People's Republic of China on the Prevention and Control of Infectious Diseases", and adopted prevention and control measures designated for class A infectious diseases. Interventional radiology has become an indispensable clinical discipline because of its unique, minimally invasive characteristics and high degree of effectiveness, playing an important role in the diagnosis and treatment of cerebrovascular, peripheral vascular, cardiovascular, nonvascular and neoplastic diseases. In the face of the complex and severe COVID-19 epidemic and the infection of numerous medical workers with the virus (8), there is a need for timely and efficient detection, prevention and emergency responses. Given the characteristics of professional diagnosis and treatment methods in interventional radiology, the Chinese Society of Interventional Radiology (CSIR) determined the need to organize multidisciplinary experts in this field in China. These experts used a series of guidelines, relevant norms and consensuses to discuss and prepare this "Expert Consensus" (9-18), which describes and summarizes the relevant protective strategies and suggestions for medical workers in the field of interventional radiology as they engage in interventional diagnosis and treatment activities against the background of novel coronavirus infection control. The aim is to provide a reference for interventional radiologists in hospitals and other medical institutions at all levels in China and worldwide.

\section{Basic principles}

In view of this major international public health emergency, all medical personnel engaged in interventional diagnosis and treatment must thoroughly understand the importance of the current prevention and control measures for the COVID-19 epidemic, feel a strong sense of urgency and responsibility for epidemic prevention, and ensure that all interventional procedures are performed in an orderly, efficient and safe manner.

\section{Interventional diagnosis and treatment principles}

Interventional diagnosis and treatment can be divided into two types according to the clinical needs: emergency intervention and elective intervention. For hospitals primarily responsible for the treatment of COVID-19, it is advisable to suspend non-emergency interventional diagnosis and treatment during the epidemic. Each medical institution can flexibly adjust to provide necessary interventional diagnosis and treatment services according to the actual characteristics of the situation, such as the epidemic control status, epidemic characteristics in the local area, and distribution of medical resources, This flexibility will include ensuring that patients with urgent, chronic or special needs receive unobstructed medical treatment or consultation by taking full advantage of "telemedicine". If the safety and effective protection of medical staff are ensured, emergency interventional diagnosis and treatment services can be provided in a standardized and orderly manner. In the interest of protecting radiological personnel and their patients, epidemic prevention and control measures should be strictly implemented throughout the entire radiology process to minimize the risk of crossinfection between doctors and patients. The specific recommendations are as follows:

For highly suspected or diagnosed COVID-19 patients Given the current epidemic, patients undergoing 


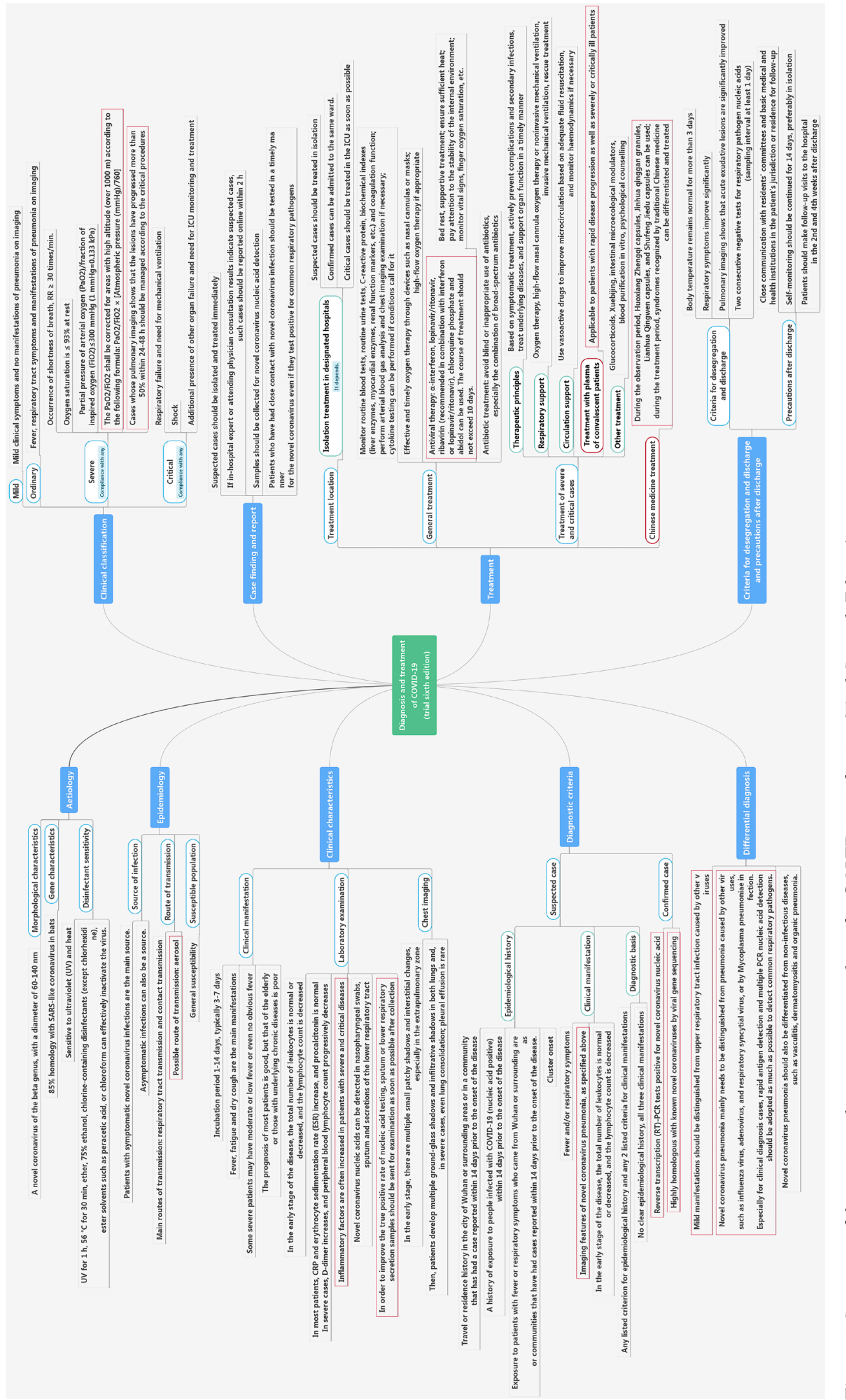

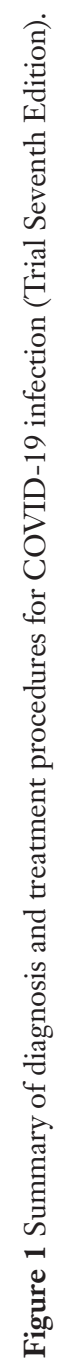


interventional surgery should be screened for COVID-19; chest radiography or CT examinations should be performed if necessary. If patients with highly suspected or diagnosed cases are in critical condition and need emergency interventions or if suspected cases need emergency interventions sooner than they can be tested, all of the following guidelines must be followed: (I) the operation should be performed in a catheter laboratory (operation room) with negative pressure and strict sterilization conditions. (II) All medical staff should use Level 3 protection, which should be approved by a COVID-19 medical coordination committee, if one has been established. For suspected patients in non-critical areas who have not yet been tested for COVID-19, if emergency interventions are required, all of the following guidelines must be followed: (I) the operation should be performed in a separate and dedicated catheter laboratory (operation room) with standard sterilization conditions. (II) All medical staff should use Level 2 or Level 3 protection, which should be approved by a COVID-19 medical coordination committee, if one has been established. After the procedure, patients diagnosed with COVID-19 should be transferred to a negative-pressure comprehensive ICU dedicated to COVID-19 patients to continue treatment. Any patient with suspected COVID-19 should be placed in a single isolation ward. The retention and management of specimens from suspected patients should be performed carefully, and the diagnosis should be confirmed as soon as possible. To ensure the stability of the patient's vital signs and maximize the benefits, all interventional procedures should follow the "four reductions principle": reducing the difficulty and time of the interventional operation, reducing the number of participants, reducing the unnecessary consumption of disposable protective equipment, and reducing the amount of equipment in the catheterization laboratory. All supplies and devices used in the interventional procedure should be available.

\section{For cancer patients}

The current understanding of COVID-19 indicates that cancer patients have an increased risk of infection, an increased risk of severe illness once infected, and an increased rate of disease progression. Prevention of infection should be given priority. The following patients may be treated if strict protective measures are taken: patients who need interventional treatment to shrink a tumour as a condition for surgery and patients who need to control the tumour volume in the short term to avoid complications associated with a large tumour burden. Treatment may be appropriately postponed for patients whose tumours are not sensitive to chemoembolization, for those receiving adjuvant treatment to further reduce the risk of recurrence and metastasis after radical surgery and for those receiving maintenance treatment for the continuous control of tumours. Patients may be appropriately switched to intravenous chemotherapy or oral treatment to avoid the increased risk of infection caused by the adverse effects of interventional treatment on the functioning of the bone marrow and important organs. It is preferable for patients to be treated at the nearest local hospital during the outbreak.

\section{For patients with acute conditions}

Acute conditions such as massive haemoptysis, acute arterial embolism, pulmonary embolism, organ rupture and bleeding, aortic aneurysm and aortic dissection rupture have an acute onset and rapid progression; patients with these conditions are considered critical. If the indication is clear, interventional treatment must be carried out immediately.

\section{For patients with acute ST-elevation myocardial infarction (STEMI)}

For patients with acute STEMI combined with haemodynamic instability or patients with life-threatening high-risk non-STEMI (NSTEMI) who need urgent revascularization, the routine treatment procedure of the chest pain centre should be followed if COVID-19 can be completely excluded; however, if the patient is first diagnosed in a hospital that does not have emergency percutaneous coronary intervention (PCI) capabilities, intravenous thrombolytic therapy should be performed $\left(3^{\text {rd }}-\right.$ generation thrombolytic drugs should be used if possible), and transportation of the patient should be reduced as much as possible. PCI can be considered if medical treatment is not effective. For patients with suspected/diagnosed cases of COVID-19 who are diagnosed with STEMI, if the onset occurred within the past $12 \mathrm{~h}$, the treatment preferred on principle is thrombolytic therapy in the hospital where the patient was initially diagnosed. After successful thrombolysis, patients should continue to be observed in the isolation ward, and they can be transferred to the cardiovascular department for elective coronary angiography if COVID-19 is subsequently excluded; if COVID-19 is diagnosed, they should be transferred to the isolation ward of the specific assigned hospital for follow-up treatment. For patients in whom thrombolysis has failed or who have contraindications to thrombolysis, clinicians must 
further evaluate the benefits of emergency PCI compared to the risks borne by both doctors and patients (risk of medical staff infection + risk of the interventional therapy). Patients should be transferred to the isolation ward for conservative treatment and further investigation of COVID-19 if the risk is greater than the benefit or if patients have been haemodynamically stable for more than $12 \mathrm{~h}$. Emergency PCI can be performed only if the benefit is significantly greater than the risk and the patient (or their guardian family members) agrees to the procedure (19).

\section{For patients with severe acute cerebrovascular diseases}

Patients with severe acute cerebrovascular diseases have an increased probability of developing severe pneumonia after infection and face an increased risk of death because of an inability to take care of themselves, disturbances of consciousness, more medical interventions, reduced preparation time before the procedure, extended operation time and extended hospitalization. Therefore, a timely diagnosis and active management strategies are needed. For "green-channel patients" with acute ischaemic stroke, CT scans of both the brain and chest should be performed simultaneously. Patients within the time window of acute ischaemic stroke should receive intravenous thrombolysis. Patients planning to undergo arterial thrombectomy should first have the possibility of COVID-19 excluded by consultation with the infectious disease (respiratory) department. Under those circumstances, intravenous thrombolysis or thrombectomy may be chosen according to the patient's condition. Those patients should not be placed in the neurological intensive care unit (NICU) after the procedure; subsequent treatment should be performed in a single room instead. Medical staff should pay attention to isolation and protection procedures to prevent crossinfection (because patients have been detained in the emergency department and have low pathogen resistance as a result of acute cerebral infarction). After infections are excluded and negative results are obtained on all tests, including three days of continuous body temperature monitoring, routine blood examinations, chest CT and COVID-19 nucleic acid tests, the patients may enter the multi-person wards. Patients with a high level of risk or suspected manifestations of COVID-19 should be quarantined in a timely manner, and physicians from relevant departments such as the respiratory department and infectious disease department should be consulted. Patients with subarachnoid haemorrhage or cerebral haemorrhage caused by arteriovenous malformation rupture often have a fever and should be rigorously screened according to the above process. For patients in whom COVID-19 cannot be completely excluded, interventional treatment should be postponed until they are found to be free from infection.

\section{Principles for prevention and control among medical staff}

(I) A "COVID-19 Prevention and Control Group" should be established in the intervention department; areas of responsibility should be divided among the members of this group, and the group should regularly hold meetings (preferably in the form of video or voice meetings);

(II) The physical condition of medical staff should be tracked in real time;

(III) Training on the prevention and control of COVID-19 should be strengthened for the medical staff affiliated with the intervention department. Training on hand hygiene should be reinforced, and staff should be reminded that wearing gloves cannot replace the practice of washing their hands with running water;

(IV) All staff should prepare, take protective measures and master the grading standard for infection prevention and control (Table 1);

(V) The monitoring of body temperature and respiratory and gastrointestinal symptoms among medical staff in the department should be strengthened;

(VI) When possible, the staff should stay home during their time off and strictly implement the provisions of the latest documents on epidemic prevention and control issued by the state, provinces or cities;

(VII) A flexible shift preparation and assignment system should be established to ensure that there are enough medical staff on duty. Overexertion of medical staff should be avoided, and staff should not work if they are ill. A nutritious diet should be provided to enhance the immunity of medical staff. The number of bedside rounds should be reduced. Bedside teaching rounds should be suspended, and the use of teaching models such as case simulations and video should be increased.

\section{Principles of patient management}

(I) For patients who are not in urgent need of medical treatment, direct-contact diagnosis and interventional treatment should be suspended, reduced or during the epidemic period; for these patients with confirmed 
Table 1 Infection prevention and control protection classification criteria

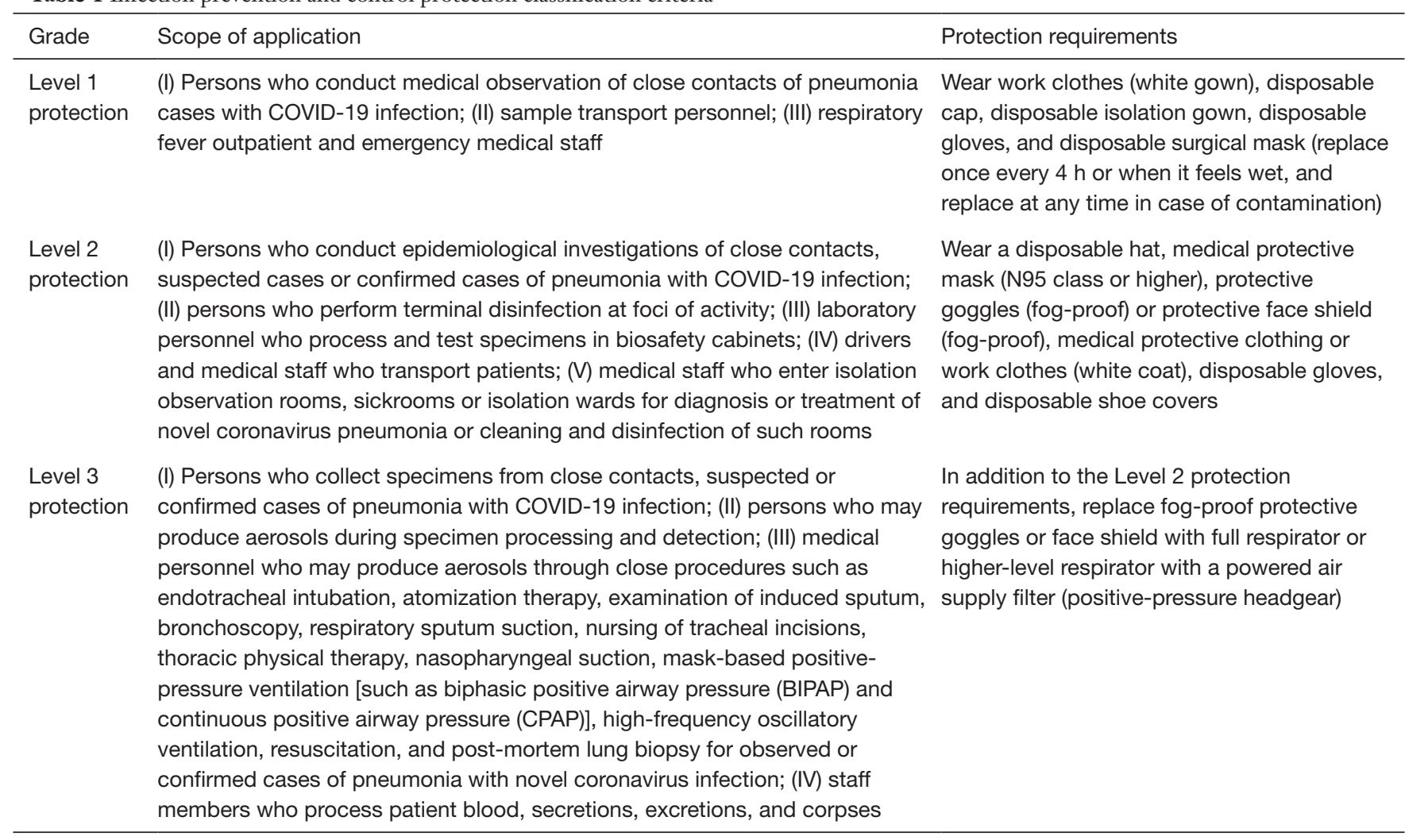

or suspected COVID-19, interventional treatment should be stopped, and guidance should be provided by telephone, Internet, and other telecommunication technologies as much as possible;

(II) For the management of ordinary patients and their families during the epidemic, the following guidelines should be implemented: (i) it is advisable to establish a sound medical pre-examination system. Interventional medical treatment should be carried out under the guidance of the COVID-19 medical coordination committee of the hospital. The monitoring of body temperature and respiratory symptoms in patients and their accompanying family members should be strengthened. (ii) Before patients are admitted for interventional diagnosis and treatment, their epidemiological contact history with regard to COVID-19 should be inquired about in detail, and patients and family members entering the hospital should wear medical masks. (iii) The waiting areas for outpatients and those needing emergency treatment should be reasonably arranged; additionally, proper ventilation management should be practised in the consultation area. Patients should not be densely concentrated while waiting, and the possibility of cross-infection should be minimized. (iv) In the outpatient service area, wards and places where crowds gather, it is necessary to strengthen the protective measures taken by individuals and groups; strengthen the education and training of patients and their accompanying family members regarding infection prevention and control; implement the standard use of protective equipment; educate and train patients and visitors in respiratory hygiene and cough etiquette (cover the mouth and nose with paper towels or elbows when coughing or sneezing); and ensure that all patients and visitors master the specifics of hand hygiene. (v) The management of hospitalized patients should be strengthened, and unnecessary outings from the hospital should be reduced. (vi) For the patients in the ward who need to be cared for, only one fixed attendant should be allowed to attend to any given patient, and the personal information of the attendant should be registered. (vii) Standardized diagnosis and treatment should be performed according to 
the requirements for prevention and control of the epidemic in hospitals. The management of febrile patients and their families should be strengthened. Those with abnormal body temperature or respiratory symptoms should be guided in a timely manner to the fever clinic for screening according to the standardized prevention, diagnosis and treatment protocols. Disinfection measures should be carried out at the activity sites once infection is diagnosed or suspected, and the staff on the COVID-19 medical coordination committee of the hospital should be informed. (viii) Doctor-patient communication should be strengthened, and mental health counselling should be actively carried out. For return visits and follow-up visits, the time limit can be appropriately adjusted, or methods such as telephone and Internet communication can be encouraged to reduce the number of times a patient visits the hospital.

(III) Management of patients with suspected or confirmed COVID-19: (i) patients with suspected or diagnosed infection should be isolated in a timely manner and guided into the isolation area by specially assigned persons according to the designated standard route. (ii) Each patient's clothes should be changed before he/ she enters the ward. Personal belongings and changed clothes should be stored in a designated place and kept according to a unified procedure by the medical institution after being disinfected in a centralized location. (iii) The patients should be instructed in the correct selection and use of masks and should correctly implement cough etiquette and hand hygiene. (iv) The management of patient visitation and attendants should be strengthened. (v) In the ward, the activities of isolated patients should be limited to reduce crossinfection. If a patient needs to leave the isolation ward or isolation area, corresponding measures should be taken; for example, the patient should wear a medical surgical mask. (vi) When a suspected or confirmed patient is discharged or transferred, he/she should change into clean clothes before leaving, and terminal disinfection of his/her contact environment should be carried out according to the "Technical Standard for Disinfection of Medical Institutions". (vii) If a patient with suspected or diagnosed COVID-19 dies, the corpse should be treated in a timely manner. The body should be wrapped in a double-layer cloth sheet and packed in a double-layer body bag. Personal items used during the patient's hospitalization should be disinfected before they are taken home.

\section{Diagnosis and treatment process for patients undergoing outpatient and emergency interventional therapy}

The nonurgent needs of patients should be managed through a wide range of channels to delay hospital visits. Patients can initially present relevant problems through online outpatient services and other relevant channels of consultation to avoid crowding the hospital and thus help maintain distance between people. Patients and their families must wear masks in the hospital. Temperature measurement should take place in the outpatient department. The interventional emergency department should be integrated into the hospital's unified process. At the entrance of the clinic and the prescreening triage station, patients and their accompanying family members should be repeatedly asked about their epidemiological risk and fever or respiratory symptoms. Blood routine test, serum IgM and IgG antibodies, and lung imaging results should be checked by interventional doctors after entering the consultation room. Once a suspected case is found, they should be transferred to a fever clinic immediately for further monitoring according to the Standard Outpatient Screening Protocol and Emergency Plan. Meanwhile, the environment the patient was in contact with should be cleaned and disinfected, and the medical staff who were in close contact with the patient should follow the Occupational Exposure Procedures (15). The Standard Outpatient Screening Protocol and Emergency Plan is provided in Figure 2.

\section{Prevention and control strategies for interventional diagnosis and treatment locations}

\section{Interventional outpatient room}

(I) Ventilation in outpatient rooms should be increased. If mechanical ventilation is used, the direction of the airflow should be controlled from the clean side to the contaminated side (17);

(II) Adequate and sufficient protective equipment should be provided for medical staff. Hand hygiene facilities such as quick-drying hand disinfectant should be provided at the entrance and exit of the clinic;

(III) Medical staff should abide by standard prevention protocols during diagnosis and treatment and should 


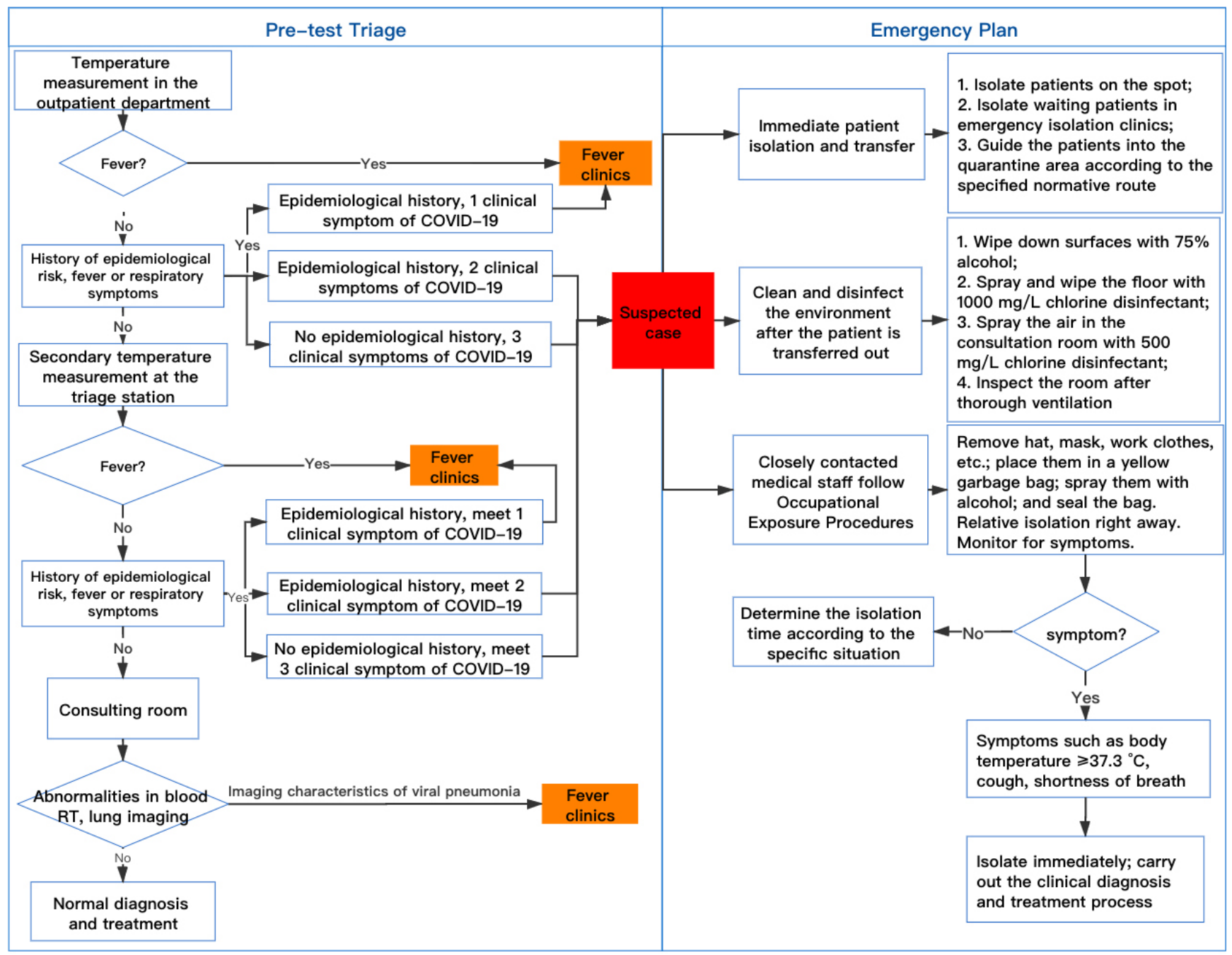

Figure 2 Standard outpatient screening protocol and emergency plan.

wear medical surgical masks or medical protective masks correctly and wash their hands or use hand sanitizer before wearing and after removing the masks;

(IV) Medical staff should master the epidemiological and clinical characteristics of novel coronavirus infection, conduct patient screenings in accordance with the diagnosis and treatment specifications, immediately take quarantine measures for patients with suspected or confirmed cases of COVID-19 and report such cases without delay;

(V) If a patient with a confirmed or suspected case of COVID-19 is found and transferred, the final disinfection should be carried out according to the Technical Specifications for the Disinfection of Medical Institutions (16);
(VI) Medical institutions should provide patients and family members with masks and instruct them on how to wear them correctly (Figure 3).

\section{Interventional ward}

(I) Emergency isolation wards should be set up for patients with suspected or confirmed cases. The relevant workflows should be established. Adequate disinfection and protective supplies for acute respiratory infections should be made available;

(II) Relevant emergency plans and work processes should be initiated once patients with suspected or confirmed cases are found in the ward. Effective isolation, treatment and referral should be implemented in a timely manner in 


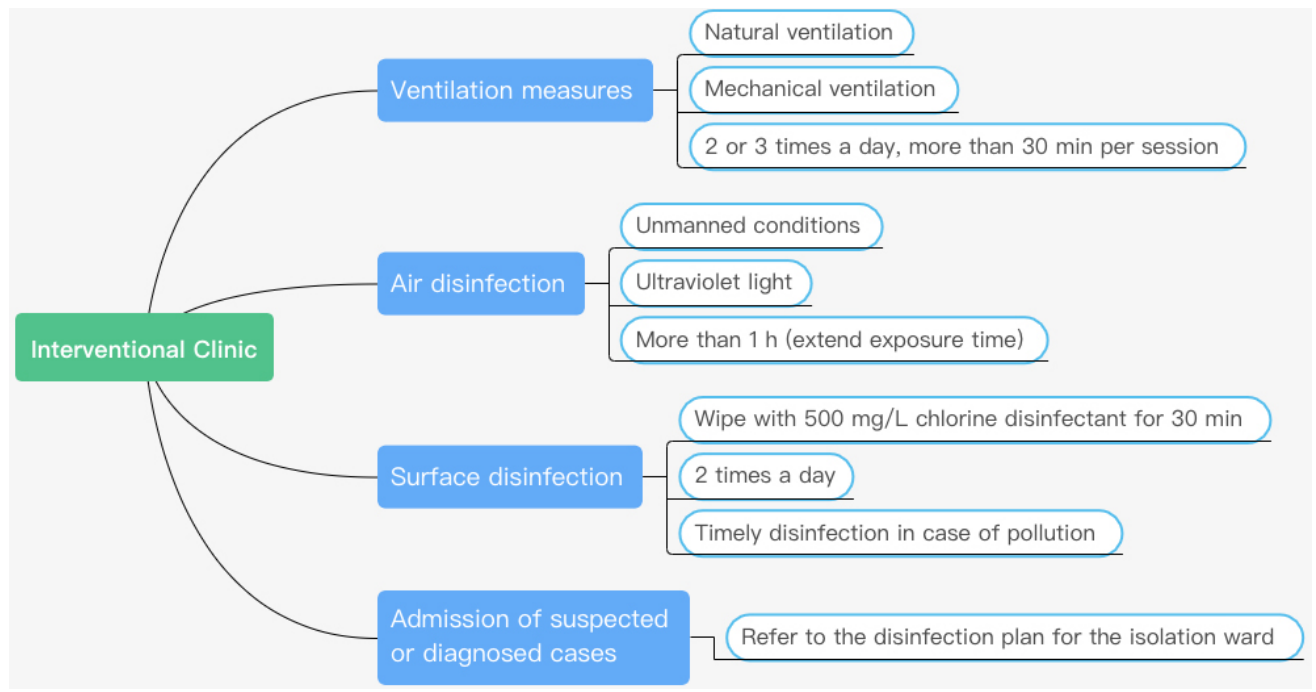

Figure 3 Interventional outpatient clinic prevention and control protocol.

accordance with the relevant guidelines;

(III) Patients with suspected or confirmed cases should be treated and cared for by specially assigned persons and should be placed in the negative-pressure ward under isolation conditions;

(IV) Patients in hospitals that do not have appropriate treatment conditions should be transferred to designated hospitals without delay. Effective isolation and treatment measures should be taken while waiting for a referral;

(V) After the patient is transferred out, the environment with which the patient came into contact should be sterilized (Figure 4).

\section{Interventional catheter laboratory}

\section{For patients with confirmed or suspected cases}

Clinical reception and preoperative signature: the patient should be accompanied by a bedside clinician and sent to the operating room through a special passage and elevator. All staff should take personal protective measures as required. Signing of preoperative forms should, in principle, be performed by family members who have no history of close contact with the patient. Patients without family members should be referred to the medical department, according to routine procedures.

Preoperative management: (I) a special interventional operating room should be dedicated. Disposable operation packs, disposable surgical instruments and related articles should be used. (II) Digitalsubtraction angiography (DSA) C-arms and flat-panel detectors should be fitted with custom-made disposable plastic film covers or disposable non-woven fabric covers, and high-pressure syringes should also be fitted with disposable plastic film covers. (III) All personnel exposed to patients with confirmed or suspected cases should adhere to the three-level protection standards.

Intraoperative management: (I) staff participating in the operation should follow strict personal disinfection and protection procedures according to the national requirements; (II) surgical personnel should put on protective equipment before entering the operating room, and staff should be forbidden to enter and exit the operating room at will; (III) it is advisable to have separate nurses stationed inside and outside the DSA operating room, and the inside personnel should not leave the operating room during surgery, while the outside personnel should not enter the operating room except under special circumstances; (IV) the required equipment and consumables should be available in the operating room prior to surgery as much as possible; (V) secondary contamination should be rigorously avoided, and if the ground and equipment are contaminated, they should be disinfected in a timely manner; and (VI) all medical waste should be discarded in a double-lined medical waste bag.

Postoperative management: (I) after the operation, staff 


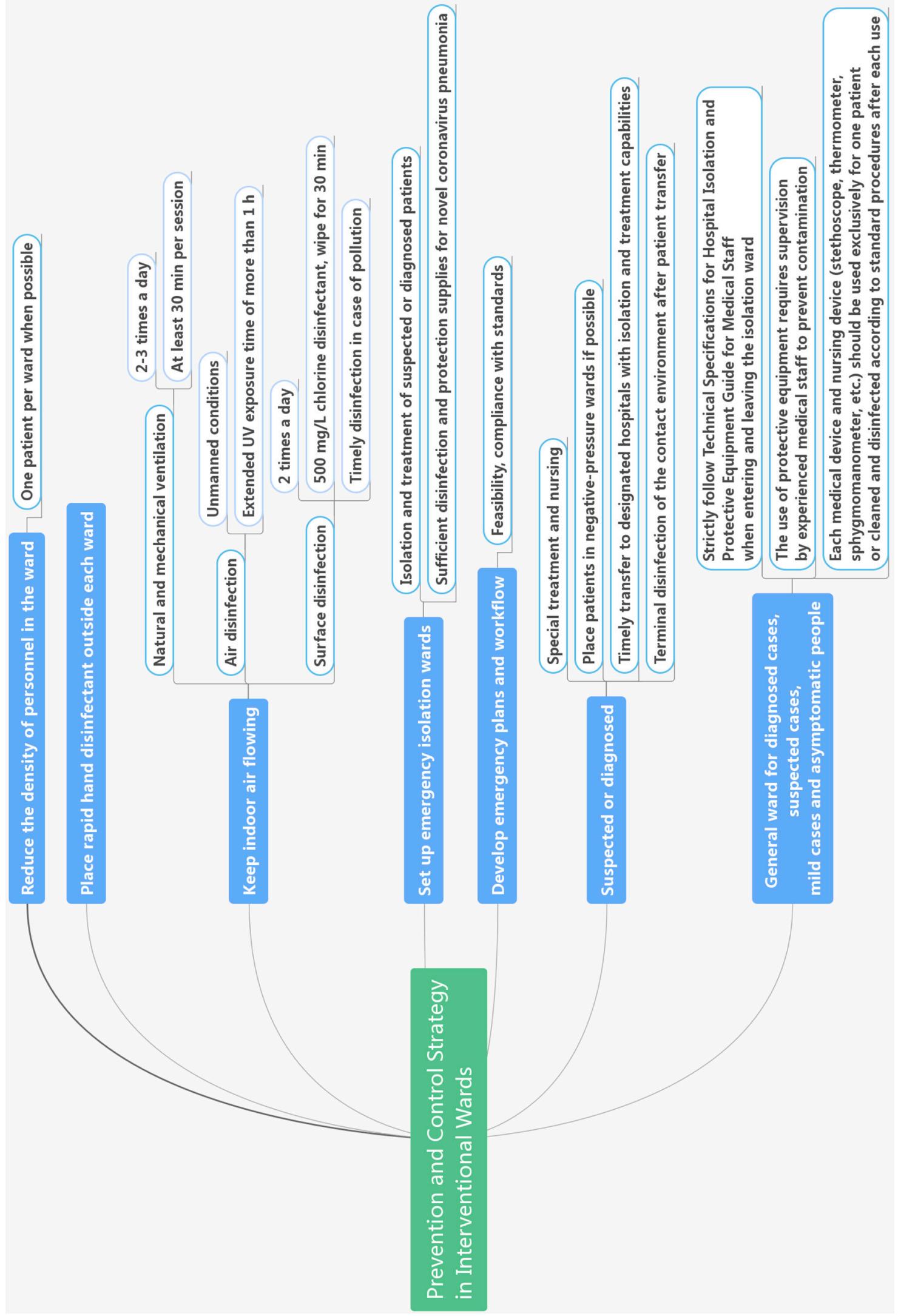

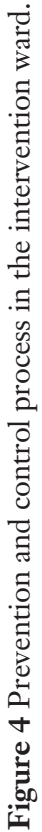


members leaving the operating room must first change their gloves and then take off their protective clothing and disinfect their hands according to the prescribed procedures. (II) After disinfecting their goggles, masks, and clothes with paper towels soaked in disinfectant, the staff should wipe them with gauze soaked in clean water, hang them on the clothes rack for drying, and place them in the clothing disinfection cabinet for disinfection if available. (III) After the operation, the participating surgical personnel should not leave the interventional operating room until they have bathed and changed their clothes. (IV) Standardized disinfection procedures should be performed in the postoperative operating room, especially on the ground, instrument table, operating table and other surfaces. (V) The laminar flow and air supply in the operating room should be closed off, and hydrogen peroxide spray should be applied for disinfection, with the room sealed for $2 \mathrm{~h}$ afterwards. After this disinfection period, the laminar flow and ventilation should be reopened. After disinfection of the negative-pressure/infection operating room, it is necessary to contact the Department of Nosocomial Infection to perform object surface and air sampling; the objects may be reused only after satisfactory results are returned. (VI) The transfer bed should be treated in the same way as the other objects in the operating room.

Personal protection for medical personnel: (I) physicians and nurses involved in the interventional therapy or exposed to patients and contaminants should be protected according to the three-level protection standards. (II) Interventional technicians, circulating nurses, and anaesthesiologists should follow secondary protection standards.

\section{For general patients \\ Patient screenings}

The patient should be asked about their epidemiological history of exposure to new coronavirus infection, clinical manifestations, and lung CT results. For patients without abnormalities, the clinician should wear a medical surgical mask when entering the operating room. If there are symptoms or signs such as fever, cough, or ground-glass opacities on lung CT, the patient should be handled according to the patient management process for patients with suspected cases of COVID-19.

\section{Preoperative preparation}

(I) In the general interventional operation room, disposable operation packs, disposable surgical instruments and related articles should be used;

(II) DSA C-arms and flat-panel detectors should be fitted with custom-made disposable plastic film covers or disposable non-woven fabric covers, and doublelayer disposable sheets should be used on the DSA surgical bed.

\section{During the intervention}

(I) Only surgeons, nurses and technicians should be allowed in the operating room;

(II) In addition to routine intraoperative cooperation by the operating nurse, the patient's secretions and vomitus should be cleaned in a timely manner.

\section{After the intervention}

(I) Once a disposable item has been used, it should be sealed in a yellow garbage bag with a timestamp and sent via the delivery channel;

(II) Reusable devices should be cleaned and disinfected according to the relevant specifications;

(III) Contaminated articles (blood pressure cuffs, etc.) should be soaked in the specified disinfectant solution and then cleaned and hung separately to dry for later use;

(IV) The door of the operating room should be opened and the room ventilated for $1 \mathrm{~h}$ after the operation; the laminar flow for laminar operations should be opened. The operating room floor, instrument table, equipment, operating table and other surfaces should be disinfected with a prescribed disinfectant.

\section{Personal protection of medical staff}

(I) The surgeon should wear a disposable work cap, medical protective mask, isolation gown, disposable latex gloves and protective goggles (fog-proof). He/ she should strictly follow surgical hand hygiene requirements;

(II) Surgical nurses and technicians should wear disposable work caps, medical protective masks, isolation gowns, disposable latex gloves, and protective goggles (fogproof) when necessary. They should strictly follow surgical hand hygiene requirements.

\section{Strengthening joint prevention and control in intervention-related disciplines}

The specialty of interventional radiology overlaps considerably with other medical specialties, such as pulmonology, oncology, vascular surgery, and neurology. Thus, the COVID-19 prevention and control team from interventional radiology should work closely with other relevant professional teams to share information and carry out interventional therapies under the unified guidance of 
the hospital's COVID-19 medical coordination committee. Finally, all medical workers should aim to protect themselves and conquer the epidemic as soon as possible.

\section{Acknowledgments}

The authors gratefully acknowledge Dr. Jingge Zhao from the clinical research and service center of Henan Provincial People's Hospital and Dr. Yiran Zhang, Dr. Junyuan Xiao and Tonglei Fang from Shanghai Jiao Tong University Affiliated Sixth People's Hospital for their contribution to the organization of the figures and tables in this article.

Funding: None.

\section{Footnote}

Conflicts of Interest: All authors have completed the ICMJE uniform disclosure form (available at http://dx.doi. org/10.21037/qims-20-516). The authors have no conflicts of interest to declare.

Open Access Statement: This is an Open Access article distributed in accordance with the Creative Commons Attribution-NonCommercial-NoDerivs 4.0 International License (CC BY-NC-ND 4.0), which permits the noncommercial replication and distribution of the article with the strict proviso that no changes or edits are made and the original work is properly cited (including links to both the formal publication through the relevant DOI and the license). See: https://creativecommons.org/licenses/by-nc-nd/4.0/.

\section{References}

1. Pan F, Ye T, Sun P, Gui S, Liang B, Li L, Zheng D, Wang J, Hesketh RL, Yang L, Zheng C. Time Course of Lung Changes On Chest CT During Recovery From 2019 Novel Coronavirus (COVID-19) Pneumonia. Radiology 2020:200370. doi: 10.1148/radiol.2020200370. [Epub ahead of print].

2. Zu ZY, Jiang MD, Xu PP, Chen W, Ni QQ, Lu GM, Zhang LJ. Coronavirus Disease 2019 (COVID-19): A Perspective from China. Radiology 2020:200490. doi: 10.1148/radiol.2020200490. [Epub ahead of print].

3. Chen N, Zhou M, Dong X, Qu J, Gong F, Han Y, Qiu Y, Wang J, Liu Y, Wei Y, Xia J, Yu T, Zhang X, Zhang L. Epidemiological and clinical characteristics of 99 cases of 2019 novel coronavirus pneumonia in Wuhan, China: a descriptive study. Lancet 2020;395:507-13.
4. Li Q, Guan X, Wu P, Wang X, Zhou L, Tong Y, Ren R, Leung KSM, Lau EHY, Wong JY, Xing X, Xiang N, Wu Y, Li C, Chen Q, Li D, Liu T, Zhao J, Li M, Tu W, Chen C, Jin L, Yang R, Wang Q, Zhou S, Wang R, Liu H, Luo Y, Liu Y, Shao G, Li H, Tao Z, Yang Y, Deng Z, Liu B, Ma Z, Zhang Y, Shi G, Lam TTY, Wu JTK, Gao GF, Cowling BJ, Yang B, Leung GM, Feng Z. Early Transmission Dynamics in Wuhan, China, of Novel CoronavirusInfected Pneumonia. N Engl J Med 2020;382:1199-207.

5. Wu Z, McGoogan JM. Characteristics of and Important Lessons From the Coronavirus Disease 2019 (COVID-19) Outbreak in China: Summary of a Report of 72314 Cases From the Chinese Center for Disease Control and Prevention. JAMA 2020. DOI: 10.1001/jama.2020.2648. [Epub ahead of print].

6. WHO. Coronavirus disease (COVID-19) technical guidance. 2020. Accessed 2020.03.31. Available online: https://www.who.int/emergencies/diseases/novelcoronavirus-2019/technical-guidance

7. WHO. Coronavirus disease (COVID-2019) situation reports. 2020. Accessed 2020.03.31. Available online: https://www.who.int/emergencies/diseases/novelcoronavirus-2019/situation-reports

8. Novel Coronavirus Pneumonia Emergency Response Epidemiology Team. The epidemiological characteristics of an outbreak of 2019 novel coronavirus diseases (COVID-19) in China. Zhonghua Liu Xing Bing Xue Za Zhi 2020;41:145-51.

9. New coronavirus pneumonia diagnosis and treatment plan (trial version 6). National Health Commission of the People's Republic of China (issued 2020.02.18). Available online: http://www.nhc.gov.cn/yzygj/s7653p/202002/8334 a8326dd94d329df351d7da8aefc2.shtml

10. Guide to New Coronavirus Infection Prevention and Control in Medical Institutions (First Edition). National Health Commission of the People's Republic of China (issued 2020.01.22). Available online: http://www.nhc.gov. cn/yzygj/s7659/202001/b91fdab7c304431eb082d67847d2 $7 \mathrm{e} 14 . \mathrm{sh} t \mathrm{ml}$

11. Guidelines for the Use of Common Medical Protective Products in the Prevention and Control of Pneumonia Due to New Coronavirus Infection (Trial). National Health Commission of the People's Republic of China. Accessed 2020.03.31. Available online: http://mpa.xinjiang. gov.cn/xjyjj/tzgg/202001/bfbe45ffc9024a8e946a03ce683 5a477.shtml

12. Chinese Society of Radiology. Radiological Diagnosis of New Coronavirus Pneumonia: Expert Recommendations 
from the Chinese Medical Association Radiology

Branch (First Edition). Chinese Journal of Radiology 2020;25:E001.

13. Chinese Society of Imaging Technology. New Coronavirus Pneumonia Imaging Hospital Infection Prevention and Control Management: Recommendations from the Imaging Technology Branch of the Chinese Medical Association (First Edition). Chinese Journal of Radiology 2020;54:E009.

14. Expert Committee on Stroke Prevention Engineering, National Health and Health Commission, Neuromedicine Professional Committee of Chinese Medical Doctor Association. Expert consensus on the prevention and control of new coronavirus infections in the field of neurointervention (first edition). Chinese Journal of Cerebrovascular Diseases 2020;17:107-12.

15. Regulation for prevention and control of healthcare associated infection in outpatient department and emergency department in healthcare facilities. In: People's Republic of China Health Industry Standard. National Health Commission of the People's Republic of China. (issued 2018.05.10). Available online: http://www.nhc.gov. cn/wjw/s9496/201805/fa830cbf8b5a4ef3a1f6615a46a35

Cite this article as: Zhu Y, He Y, Wang Z, Jin Z, Zheng C, Sun G, Shi H, Li H, Li X, Xiang H, Lu L, Zhou S, Li T, Li X, Zhu H, Huang M, Duan F, Shao H, An T, Cheng Y; Chinese Society of Interventional Radiology. Chinese Society of Interventional Radiology Expert Consensus on the prevention and control of COVID-19 in interventional radiology procedures (first edition). Quant Imaging Med Surg 2020;10(5):1045-1057. doi: 10.21037/qims.2020.04.11 0a0.shtml.

16. Regulation of disinfection technique in healthcare settings. In: People's Republic of China Health Industry Standard. Ministry of Health of the People's Republic of China. 2012. Accessed 2012.04.05. Available online: http://www. nhc.gov.cn/wjw/s9496/201204/54510.shtml

17. Regulation for prevention and control of healthcare associated infection of airborne transmission disease in healthcare facilities. National Health and Family Planning Commission of PRC. (issued 2016.12.27). Available online: http://www.nhc.gov.cn/wjw/s9496/201701/7e0e8fc672584 3 aabba8f841f2f585d2.shtml

18. Liang B, Zheng CS, Xiao SP. Strategies and recommendations for prevention and control of new coronavirus infection in interventional department. Journal of Interventional Radiology 2020;29:119-23.

19. Diagnosis and treatment process of acute myocardial infarction in the prevention and control of new coronavirus (2019-nCoV) Chinese experts suggest (first edition). Shanghai Medical Doctor Association (issued 2020.02.05) Available online: https://www.shmda.org.cn/Web/Article/ WorkingDetail.aspx?Article=75044143998F4A648EFB83 C950FD07CD 